ORIGINAL ARTICLE

\title{
Health related quality of life in disorders of defecation: the Defecation Disorder List
}

\author{
W P Voskuijl, H J van der Zaag-Loonen, I J G Ketel, M A Grootenhuis, B H F Derkx, M A Benninga
}

Arch Dis Child 2004;89:1124-1127. doi: 10.1136/adc.2003.037465

See end of article for authors' affiliations

Correspondence to: Dr W P Voskuiil, Department of Paediatric Gastroenterology and Nutrition, Room C2-312, Academic Medical Center, Meibergdreef 9, $1105 \mathrm{AZ}$ Amsterdam, Netherlands; w.p.voskuij|@amc.uva.nl

Accepted 25 February 2004

\begin{abstract}
Background: Constipation and encopresis frequently cause problems with respect to emotional wellbeing, and social and family life. Instruments to measure Health Related Quality of Life (HRQoL) in these disorders are not available.

Methods: A disease specific HRQoL instrument, the "Defecation Disorder List" (DDL) for children with constipation or functional non-retentive faecal soiling (FNRFS) was developed using accepted guidelines. For each phase of the process, different samples of patients were used. The final phase of development included 27 children. Reliability was assessed in two ways: internal consistency of domains with Cronbach's alpha, and test-retest reliability with intra-class correlation coefficients (ICC). To assess validity, comparable items and domains were correlated with Tacqol, a generic HRQoL instrument for children (TNO-AZL).

Results: In the final phase of the development, 27 children completed the instrument. It consisted of 37 items in four domains. The response rate was $96 \%$. Reliability was good for all domains, with Cronbach's alpha values ranging from 0.61 to 0.76 . Measures of test-retest stability were good for all four domains with ICCs ranging from 0.82 to 0.92 . Validity based on comparison with the Tacqol instrument was moderate.

Conclusion: The DDL is promising as a measure of $\mathrm{HRQ}$ oL in childhood defecation disorders.
\end{abstract}

\section{METHODS}

The HRQoL questionnaire was developed using accepted guidelines. ${ }^{14}$

\section{Phase 1: Defining population and objectives for development}

Patients with constipation or FNRFS (7-15 years old) referred to our tertiary care centre by school doctors, general physicians, and paediatricians were eligible. They were included in the study if:

(1) They met at least two of four criteria for paediatric constipation: $^{15}$ fewer than three bowel movements per week (without laxatives); encopresis at least twice weekly; intermittent passage of very large stools (every 7-30 days); palpable abdominal or rectal mass

(2) They fulfilled the criteria for FNRFS: encopresis at least twice weekly in the absence of constipation.

All had symptoms for at least six months.

\section{Phase 2: Item generation}

Item generation was performed through group discussion among paediatric gastroenterologists and psychologists. A list of 102 items covering physical, social, emotional, and treatment issues was generated.

Phase 3: Item reduction, phrasing, and formatting The goals of item reduction are to eliminate redundant or inappropriate items, to include manageable numbers of questions, and to create a valid scale. Forty three patients (able to read and understand Dutch) were asked to rate the importance to their lives of each of the 102 items using a four

Abbreviations: FNRFS, functional non-retentive faecal soiling; HRQoL, health related quality of life; ICC, intra-class correlation coefficients; Tacqol, TNO-AZL Children's Quality of Life Questionnaire 
point Likert scale. This ranged from least important ( 1 point) to most important (4 points). Initially they completed the questionnaire unsupervised, but if they did not understand items, they could receive help from a parent. Based on these scores, items were ranked, and the 33 most important for the total group, as well as for age and sex, were selected for inclusion. In addition, eight relevant general items were included from the Impact HRQoL instrument for children with inflammatory bowel disease. ${ }^{8}$

The questionnaire was also tested by one of the investigators (IJGK) using the Question Appraisal System (QAS-99) (Willis GB and Lessler JT, Research Triangle Institute, Rockville, USA) to identify problems of phrasing.

Five children completed the questionnaire and were then interviewed. This was to ensure that they understood the content and that all interpreted the question similarly. This led to some changes in phrasing. These children were also asked if they considered that important items were missing, but none were identified.

Questions were phrased in the first person. Answers were based on a five point Likert scale. The instrument now contained 41 items within four domains: constipation related, emotional functioning, social functioning, and treatment/interventions.

\section{Phase 4: Pilot testing of the questionnaire}

A further group of 26 children (18 boys) completed the questionnaire to ensure feasibility and to identify redundant items. These suffered from constipation with encopresis $(n=18)$, constipation alone $(n=3)$, and FNRFS $(n=5)$.

Spearman rank order correlation coefficients were calculated between all items to identify redundant items. This was considered if the coefficient was $>0.6$ and/or if items were of comparable content.

\section{Phase 5: Modifications of pre-final instrument}

Three of 41 items closely correlated and/or were of comparable content; they were excluded, leaving 38 items.

\section{Phase 6: Reliability and validity}

All children involved in the final testing phase kept a diary to record stool and encopresis frequency. They underwent a toilet training regime (three times daily) with a reward system. The constipated patients received laxative treatment.

The DDL and the generic Tacqol instrument (TNO-AZL) ${ }^{16}$ were used in this phase. The latter examines health status in recent weeks and the emotional response to problems. Tacqol uses a four point Likert scale and contains seven domains, each with eight items: physical complaints, motor functioning, autonomy, cognitive functioning, social functioning, and positive and negative emotions.

Table 1 Patient characteristics at time of completing the $\mathrm{DDL}$

\begin{tabular}{ll}
\hline Characteristic & No. \\
\hline No. of patients & 27 \\
Median years (range) & 10.7 (7.3-14.3) \\
No. of boys & 18 \\
No. with constipation and encopresis & 18 \\
No. with constipation only & 4 \\
No. with FNRFS & 5 \\
Encopresis, episodes/week; median (range) & $2(0-8.5)$ \\
Duration of previous treatment in months; & $33(4.5-132)$ \\
median (range) & \\
Duration of previous symptoms in years; & $6.4(1.4-12.4)$ \\
median (range) &
\end{tabular}

Table 2 Internal consistency (Cronbach's $\alpha$ ) and ICCs

\begin{tabular}{lcll}
\hline Domains & No. of items & Cronbach's $\boldsymbol{\alpha}$ & ICCs \\
\hline Constipation related & 3 & 0.65 & 0.92 \\
Emotional functioning & 13 & 0.76 & 0.89 \\
Social functioning & 11 & 0.63 & 0.82 \\
Treatment/interventions & 10 & 0.61 & 0.87 \\
Total instrument & 37 & & 0.87 \\
\hline
\end{tabular}

After consenting to participate, patients received the two questionnaires. If no response was received within two weeks, they were contacted to encourage participation. Two weeks later the DDL questionnaire was again sent to the participants. Again, if necessary the family were contacted.

This final phase included 28 subjects ( 19 boys), including children with constipation with encopresis $(n=19)$, constipation without encopresis $(n=4)$ and FNRFS $(n=5)$.

\section{Statistical analysis}

Reliability

First, "floor and ceiling effects" were examined. If a high proportion scored the highest (ceiling) or lowest possible (floor) score on an item, this item was considered to be of limited value in detecting change over time. ${ }^{17}$ Reliability was assessed by examining "internal consistency" and "testretest reliability". Internal consistency (or homogeneity), referring to the extent to which the items in a domain assess the same characteristic, was measured using Cronbach's alpha. ${ }^{18}$ Homogeneity was considered satisfactory if the Cronbach's alpha value was $>0.6$ and excellent if $>0.9$. Reliability was assessed by repeat completion of the questionnaire after two weeks. It was assumed that symptoms would be unchanged over this period. For test-retest analysis, intra-class correlation coefficients (ICCs) were calculated for each item and each domain to assess the correlation between scores while correcting for systematic differences in scores.

Validity

"Validity" is defined as the extent to which the instrument measures what it is intended to measure. In developing the instrument, validity was tested in three ways. "Content validity" was tested during the early phases by ensuring that no issues of importance were omitted. "Construct validity" was tested by examining correlations with comparable items and domains in the Tacqol instrument. If this disease related instrument was measuring HRQoL, it should correlate with the generic instrument. Since disease specific instruments are more likely to detect small changes, such correlations would not normally be very high. A Spearman rank order coefficient between 0.4 and 0.6 was considered acceptable. "Discriminatory validity" was assessed based on the hypothesis that

Table 3 Correlations of the DDL with overlapping items and domains in the Tacqol generic instrument

\begin{tabular}{lll}
\hline DDL item & Tacqol item & Spearman rank order \\
\hline Stomach aches & Stomach item & 0.74 \\
Angry to have problem & Feeling angry & 0.17 \\
Being happy & Feeling happy & 0.55 \\
Being happy & Feeling pleased & 0.55 \\
\hline DDL domain & Tacqol domain & Spearman rank order \\
\hline Emotional functioning & Positive emotions & 0.03 \\
Emotional functioning & Negative emotions & 0.19 \\
Social functioning & Social functioning & 0.14 \\
\hline
\end{tabular}


Table 4 Discriminant validity of the DDL in comparing low frequency $(\leqslant 2$ times per week; group 1) and high frequency (>2 times per week; group 2) encopresis

\begin{tabular}{lllll}
\hline Domains & $\begin{array}{l}\text { Domain score } \\
\text { Group 1 }\end{array}$ & $\begin{array}{l}\text { Domain score } \\
\text { Group 2 }\end{array}$ & p value & $\begin{array}{l}\text { Mean difference } \\
\text { (95\% Cl) }\end{array}$ \\
\hline Constipation related & 10.13 & 10.08 & 0.95 & $0.05(-1.7$ to 1.8) \\
Emotional functioning & 25.73 & 26.75 & 0.69 & $1.02(-6.2$ to 4.2$)$ \\
Social functioning & 22.60 & 26.50 & 0.08 & $3.90(-8.3$ to 0.5$)$ \\
Treatment/interventions & 22.27 & 28.67 & 0.02 & $6.40(-11.8$ to -1.0$)$ \\
Total instrument & 87.00 & 97.92 & 0.09 & $10.92(-23.8$ to 2.0) \\
\hline Independent Student's $t$ test used to compare mean domain scores between groups. \\
\hline
\end{tabular}

HRQoL in those with very frequent encopresis would be lower than with lower frequency. Therefore, the mean domain scores in those with encopresis $\leqslant 2$ times weekly, and those with $>2$ times weekly were compared, using the independent Student's $t$ test.

\section{RESULTS}

Table 1 shows patient characteristics. Overall 27 of the 28 subjects completed the first and second questionnaires, and each completed all items.

\section{Reliability: internal consistency and test-retest reliability (table 2 )}

Cronbach's $\alpha$ values ranged from 0.61 to 0.76 , indicating good consistency within the domains. ICCs ranged from 0.82 to 0.92 , with an overall value for the questionnaire of 0.87 . This indicates good to excellent reproducibility.

Four items $(18,31,37$, and 42$)$ of the DDL suffered from floor effects, with $70 \%$ choosing the highest possible score (lowest in terms of HRQoL). Three of these items were in the social functioning domain. Only one item had to be removed from the final instrument because it did not contribute to homogeneity (Cronbach's alpha) in any of the four domains. Therefore, the final instrument consisted of 37 items.

\section{Validity (table 3)}

The correlation between the DDL and the generic instrument with Spearman rank order coefficients was relatively low and ranged from 0.03 to 0.74 . The domains related to "positive emotions" and "social functioning" showed an especially low correlation. With respect to discriminatory validity, only the treatment domain differed significantly between the groups (table 4). Subgroup analysis showed that in the male subgroup a statistically significant difference between those with a high and low encopresis frequency persisted in the treatment domain, but no differences were found in the other domains. In the female subgroup no domains were significantly different when comparing those with high and low encopresis frequency.

\section{DISCUSSION}

This study describes the development of the first HRQoL instrument specifically for children with constipation and functional non-retentive faecal soiling. The questionnaire was developed using accepted standards for the development of such instruments. The final questionnaire consisted of 37 items in four domains. It appears to provide a valid and reliable instrument. The correlation with an established generic HRQoL instrument (Tacqol) was relatively low. The response rate to the questionnaire was excellent (96\%). Patients filled in all items of the questionnaire and so it appears to be satisfactory in terms of patient acceptability.

Establishing the validity of an HRQoL instrument is difficult given the absence of gold standards. ${ }^{19}$ Validity of the DDL with respect to construct validity was moderate. Comparable items and domains correlated moderately well with the Tacqol instrument. This could be explained by subtle differences in wording. For example, an item from the Tacqol asks about "feeling angry" while an item from the DDL asks about only "feeling angry at having this disorder"; these items had a correlation coefficient of 0.17 (table 3).

Childhood constipation is common and about 30\% of patients continue to have symptoms at 16 years of age; ${ }^{5}$ it is therefore important to have available an HRQoL instrument. Generic quality of life instruments are considered unsatisfactory, and disease specific instruments are to be preferred. $^{20-22}$

Many children with constipation suffer from encopresis. This often has a profoundly negative effect on the social and emotional aspects of life. Until now there have been few studies formally evaluating the impact of these conditions on quality of life. Two reports on children following surgical correction for anorectal malformations and Hirschsprung's disease have reported that faecal soiling has a major impact on the HRQoL. ${ }^{12} 23$

The sensitivity of this instrument and its ability to detect changes over time require further evaluation using larger samples of patients. Currently the questionnaire is being translated into other languages, but these translations will require formal evaluation.

Apart from differences in wording, the low correlation between the Tacqol and DDL instruments implies that the instruments are not measuring the same thing. Either the Tacqol is unsatisfactory or our instrument is not valid. Thus if a patient with encopresis fills out an HRQoL questionnaire that does not address this specific symptom, the final score will be relatively lower than with a disease specific instrument.

Validity with respect to the discriminatory capabilities of the instrument was not as good as had been hoped. The confidence intervals of the domain scores comparing those with a low and high frequency of encopresis were wide (table 4). The mean differences between these groups were large for both the "social functioning" and "treatment/ interventions" domains. The latter difference was significantly different $(p=0.02)$. Comparing the total DDL scores of the two groups, the mean difference was 10.9 (97.9 minus 87.00).

Four items in the HRQoL showed significant "floor effects", so that they could not be expected to detect changes over time. Three of these were in the social domain, but they may nevertheless provide important information in other respects.

In summary, the DDL is a promising disease specific HRQoL instrument for children between 7 and 15 years of age with constipation or FNRFS. The ultimate purpose of this instrument is in the assessment of therapy and its impact on HRQoL. It now requires further study in a larger patient population. 


\section{Authors' affiliations}

W P Voskuijl, H J van der Zaag-Loonen, I J G Ketel, B H F Derkx,

M A Benninga, Department of Paediatric Gastroenterology and

Nutrition, Emma Children's Hospital/Academic Medical Center,

Amsterdam, Netherlands

M A Grootenhuis, Psychosocial Department, Emma Children's Hospital/

Academic Medical Center, Amsterdam, Netherlands

\section{REFERENCES}

1 Loening-Baucke VA, Younoszai MK. Abnormal anal sphincter response in chronically constipated children. J Pediatr 1982;100:213-18.

2 van der Plas RN, Benninga MA, Buller HA, et al. Biofeedback training in treatment of childhood constipation: a randomised controlled study. Lancet 1996:348:776-80.

3 Brooks RC, Copen RM, Cox DJ, et al. Review of the treatment literature for encopresis, functional constipation, and stool-toileting refusal. Ann Behav Med $2000 ; 22: 260-7$

4 Staiano A, Andreotti MR, Greco L, et al. Long-term follow-up of children with chronic idiopathic constipation. Dig Dis Sci 1994;39:561-4.

5 van Ginkel R, Reitsma JB, Buller HA, et al. Childhood constipation: longitudinal follow-up beyond puberty. Gastroenterology 2003; 125:357-63.

6 Rasquin-Weber A, Hyman PE, Cucchiara S, et al. Childhood functional gastrointestinal disorders. Gut 1999;45(suppl 2):60-8.

7 Rockney RM, McQuade WH, Days AL. The plain abdominal roentgenogram in the management of encopresis. Arch Pediatr Adolesc Med 1995; 149:623-7

8 Loonen HJ, Grootenhuis MA, Last BF, et al. Measuring quality of life in children with inflammatory bowel disease: the impact-II (NL). Qual Life Res 2002; 11:47-56.

9 Asmussen L, Olson LM, Grant EN, et al. Reliability and validity of the Children's Health Survey for Asthma. Pediatrics 1999;104:e71.
10 Hoare $\mathbf{P}$, Russell M. The quality of life of children with chronic epilepsy and their families: preliminary findings with a new assessment measure. Dev Med Child Neurol 1995;37:689-96.

11 Calaminus G, Kiebert G. Studies on health-related quality of life in childhood cancer in the European setting. Int J Cancer 2002;12:83-6.

12 Bai Y, Yuan Z, Wang W, et al. Quality of life for children with faecal incontinence after surgically corrected anorectal malformation. J Pediatr Surg 2000;35:462-4.

13 Rockwood TH, Church JM, Fleshman JW, et al. Faecal Incontinence Quality of Life Scale: quality of life instrument for patients with faecal incontinence. Dis Colon Rectum 2000;43:9-16.

14 Loonen HJ, Derkx BH, Otley AR. Measuring health-related quality of life of pediatric patients. J Pediatr Gastroenterol Nutr 2001;32:523-6.

15 Loening-Baucke V. Modulation of abnormal defecation dynamics by biofeedback treatment in chronically constipated children with encopresis. J Pediatr 1990;116:214-22.

16 Theunissen NC, Vogels TG, Koopman HM, et al. The proxy problem: child report versus parent report in health-related quality of life research. Qual Life Res 1998:7:387-97.

17 Korevaar JC, Merkus MP, Jansen MA, et al. Validation of the KDQOL-SF: a dialysis-targeted health measure. Qual Life Res 2002;11:437-47.

18 Cronbach L. Coefficient alpha and the internal structure of tests. Psychometrika 1951;16:297-334.

19 Eiser C, Morse R. Issues in measuring QoL in children. Health Technology Assessment 2001;58(4):9-19.

20 Loonen HJ, Grootenhuis MA, Last BF, et al. Quality of life in paediatric inflammatory bowel disease measured by a generic and a disease-specific questionnaire. Acta Paediatr 2002;91:348-54.

21 Guyatt GH, King DR, Feeny DH, et al. Generic and specific measurement of health-related quality of life in a clinical trial of respiratory rehabilitation. J Clin Epidemiol 1999;52:187-92.

22 Bradley C. Importance of differentiating health status from quality of life. Lancet 2001;357:7-8.

23 Bai $Y$, Chen $\mathrm{H}$, Hao J, et al. Long-term outcome and quality of life after the Swenson procedure for Hirschsprung's disease. J Pediatr Surg 2002;37:639-42.

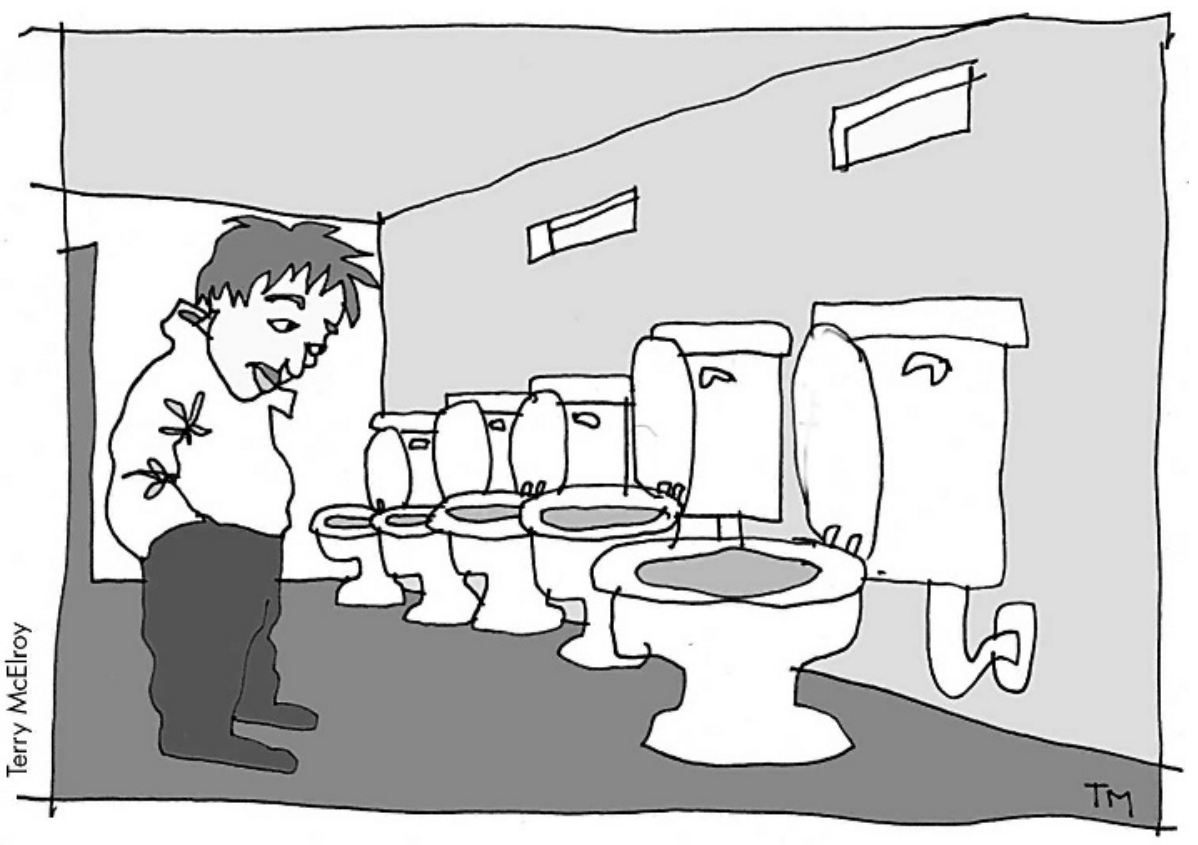

